\title{
Anthocyanin regulatory and structural genes associated with violet flower color of Matthiola incana
}

\author{
Latifa Nuraini ${ }^{1} \cdot$ Yukiko Ando $^{2} \cdot$ Kentaro Kawai $^{2} \cdot$ Fumi Tatsuzawa $^{4} \cdot$ Kotomi Tanaka $^{3} \cdot$ Masaki Ochiai $^{1,5}$. \\ Katsumi Suzuki $^{1,2,3,6} \cdot$ Verónica Aragonés $^{7} \cdot$ José-Antonio Daròs ${ }^{7} \cdot$ Takashi Nakatsuka $^{1,2,3,6} \mathbb{C}$
}

Received: 30 August 2019 / Accepted: 29 January 2020 / Published online: 8 February 2020

○) Springer-Verlag GmbH Germany, part of Springer Nature 2020

\begin{abstract}
Main conclusion MiMYB1 and MibHLH2 play key roles in anthocyanin biosynthesis in Matthiola incana flowers. We established a transient expression system using Turnip mosaic virus vector in $M$. incana.
\end{abstract}

\begin{abstract}
Garden stock (Matthiola incana (L.) R. Br.) is a popular flowering plant observed from winter to spring in Japan. Here we observed that anthocyanin accumulation in 'Vintage Lavender' increased with flower development, whereas flavonol accumulation remained constant throughout flower development. We obtained five transcription factor genes, MiMYB1, MibHLH1, MibHLH2, MiWDR1, and MiWDR2, from M. incana floral cDNA contigs. Yeast two-hybrid analyses revealed that MiMYB1 interacted with MibHLH1, MibHLH2, and MiWDR1, but MiWDR2 did not interact with any transcription factor. Expression levels of MiMYB1 and MibHLH2 increased in petals during floral bud development. Their expression profiles correlated well with the temporal profiles of MiF3'H, MiDFR, MiANS, and Mi3GT transcripts and anthocyanin accumulation profile. On the other hand, MibHLHI was expressed weakly in all organs of 'Vintage Lavender'. However, high expression levels of $\mathrm{MibHLH1}$ were detected in petals of other cultivars with higher levels of anthocyanin accumulation than 'Vintage Lavender'. MiWDR1 and MiWDR2 maintained constant expression levels in petals during flower development and vegetative organs. Transient MiMYB1 expression in 1-month-old $M$. incana seedlings using a Turnip mosaic virus vector activated transcription of the endogenous anthocyanin biosynthetic genes MiF3'H, MiDFR, and MiANS and induced ectopic anthocyanin accumulation in leaves. Therefore, MiMYB1 possibly interacts with MibHLH2 and MiWDR1, and this trimeric protein complex activates the transcription of anthocyanin biosynthetic genes in $M$. incana flowers. Moreover, MibHLH1 acts as an enhancer of anthocyanin biosynthesis with the MiMYB1-MibHLH2-MiWDR1 complex. This study revealed the molecular mechanism involved in the regulation of anthocyanin accumulation levels in $M$. incana flowers.
\end{abstract}

Keywords Basic helix-loop-helix · Garden stock · R2R3MYB · Turnip mosaic virus · WD40 repeat

Latifa Nuraini, Yukiko Ando, Kentaro Kawai contributed equally to this work.

Electronic supplementary material The online version of this article (https://doi.org/10.1007/s00425-020-03351-z) contains supplementary material, which is available to authorized users.

Takashi Nakatsuka

nakatsuka.takashi@shizuoka.ac.jp

1 The United Graduate School of Agricultural Science, Gifu University, 1-1 Yanagido, Gifu-shi, Gifu 501-1193, Japan

2 Graduate School of Integrated Science and Technology, Shizuoka University, 836 Ohya, Suruga-ku, Shizuoka 422-8529, Japan

3 Faculty of Agriculture, Shizuoka University, 836 Ohya, Suruga-ku, Shizuoka 422-8529, Japan
4 Faculty of Agriculture, Iwate University, 3-18-8 Ueda, Morioka 020-8550, Japan

5 Faculty of Applied Biological Sciences, Gifu University, Yanagido 1-1, Gifu 501-1193, Japan

6 College of Agriculture, Academic Institute, Shizuoka University, 836 Ohya, Suruga-ku, Shizuoka 422-8529, Japan

7 Instituto de Biología Molecular y Celular de Plantas (Consejo Superior de Investigaciones Científicas-Universitat Politècnica de València), 46022 Valencia, Spain 


\section{Introduction}

Garden stock (Matthiola incana (L.) R. Br.), which belongs to the Brassicaceae family, is an important annual ornamental plant worldwide (Dole and Wilkins 2005). Other members of Brassicaceae include Lunaria in addition to some of the crucial vegetable crops worldwide such as broccoli, brussels sprouts, cabbage, horseradish, kale, kohlrabi, mustard, radish, rutabaga, and turnip (Dole and Wilkins 2005). The earliest record of $M$. incana describes it as a hoary biennial with purple and white flowers with blackish green seeds (Saunders 1928). Genetic variation is observed at flowering time, which could be attributed to differential sensitivity to low temperature and day length in addition to varying lengths of the juvenile phase (Hisamatsu et al. 2000). Most currently known cultivars are annual, and their low temperature requirement for flowering is generally more stringent for late-flowering cultivars rather than for early-flowering cultivars. $M$. incana cultivars are used as cut and pot flowers, and they bloom in winter and spring in Japan.

Up to 14 commercial colors of $M$. incana are marketed; these range from pure white to lavender, purple, blue, red, and pale yellow (Dole and Wilkins 2005). Anthocyanins, particularly pelargonidin and cyanidin derivatives, are responsible for the flower color, but delphinidin derivatives are not detected in the investigated commercial cultivars of $M$. incana (Tatsuzawa et al. 2012). Wild $M$. incana has petal color ranging from purple to violet (Saunders 1928), with cyanidin 3-[2-(2-(sinapoyl)-xylosyl)-6(feruloyl)-glucoside]-5-[6-(malonyl)-glucoside] as the major pigment (Saito et al. 1995). The flower color diversity in $M$. incana cultivars is thought to be caused by a slightly different modification of the major anthocyanins derived from pelargonidin or cyanidin and differences in anthocyanin levels (Tatsuzawa et al. 2012).

The study by Saunders contributed substantially to the understanding of the genetics of $M$. incana, and it was speculated that the flower color of $M$. incana was under the control of four genes $(C, R, B$, and $V)$. $C$ and $R$ are both required for anthocyanin production in flowers (Saunders 1928). $B$ converts red anthocyanins to blue, and $V$ is linked to the difference between the pure color $V$ and impure color $v$ (Crane and Lawrence 1947; Saunders 1928). The colors resulting from the combination of the four genes are as follows: purple, $C R B V$; plum, $C R B v$; red, $C R b V$; copper, $C R b v$; and white, other genotypes. Dilute flower colors (e.g., rose, pale marine, and azure) are dominant over deep colors (e.g., carmine, marine, and purple). In addition to the abovementioned genes, $W$ is responsible for the difference between the white color $W$ and cream color $w$ (Crane and Lawrence 1947; Saunders 1928). Because $W$ is not entirely dominant, the heterozygote has an intermediate sulfur color.
M. incana has been applied as a model system for studies on anthocyanin biosynthesis (Forkmann 1993; Seyffert 1971). Studies have explored three loci, $B, L$, and $U$, which are involved in the enzyme step involving hydroxylation at the $3^{\prime}$ position (Forkmann 1980), glucosylation at the 5' position (Teusch et al. 1986), and acylation of anthocyanin (Teusch et al. 1987), respectively. Hemleben et al. (2004) also reported on three white-flowered mutant lines, which were influenced by $e, f$, and $g$. $F$ encodes a chalcone synthase (CHS) (Hemleben et al. 2004; Rail and Hemleben 1984; Spribille and Forkmann 1981). The white-flowered and glabrous $e$ mutant exhibits one nucleotide change, leading to a direct amino acid substitution in the WD motif (W158R) of TRANSPARENT TESTA GLABRA1 (TTG1, Dressel and Hemleben 2009). Transient expression of two basic helix-loop-helix (bHLH) genes, i.e., MYC146 and GLABRA 3 (GL3), derived from Arabidopsis thaliana, can activate anthocyanin biosynthesis in white-flowered $g$ mutant (Ramsay et al. 2003). Therefore, both $G$ and $E$ have been demonstrated to exert a pleiotropic action on the formation of leaf hairs (Forkmann 1993).

Although the genetic analysis of $M$. incana flower colors has been performed extensively, molecular genetics-based information on the flowers is yet to be reported. Recently, Nakatsuka and Koishi (2018) identified the deletion mutation alleles of $M$. incana AGAMOUS $(M i A G)$, which have been known as doubled flower $s$ locus in genetic analysis (Saunders 1928). Numerous molecular biological studies on anthocyanin biosynthesis have been conducted using model plants such as A. thaliana (Saito et al. 2013), cabbage (Brassica oleracea var. capitate; Yuan et al. 2009), kale (B. oleracea var. acephala; Zhang et al. 2012), cauliflower (B. oleracea var. botrytis; Chiu et al. 2010), B. rapa (Li et al. 2012), and radish (Raphanus sativus; Lim et al. 2016, 2017), which also belong to Brassicaceae. The MYB-bHLH-WDR (MBW) complex, which is composed of three proteins [i.e., R2R3-MYB, bHLH, and WD40 repeat (WDR)] regulates anthocyanin biosynthesis (Allan and Espley 2018; Nakatsuka et al. 2014; Xu et al. 2015). In $M$. incana flower pigmentation, $b H L H$ and $W D R$ could be correlated with $G$ and $E$, respectively (Dressel and Hemleben 2009; Ramsay et al. 2003). RNA-seq based on next-generation sequencing (NGS) has been used to identify several genes associated with key floral traits in horticultural plants in cases wherein genome information is lacking (Nakatsuka et al. 2019; Sasaki et al. 2017; Suzuki et al. 2016; Zhao et al. 2014). Chen et al. (2018) reported on differentially expressed genes (DEGs) among three flower color varieties: white, light purple, rose bengal $M$. incana flowers. They successfully identified $\mathrm{CHS}$, chalcone isomerase (CHI), flavanone 3-hydroxylase $(\mathrm{F} 3 \mathrm{H})$, anthocyanidin synthase (ANS), UGT78D2, and flavonol synthase 1 (FLS1) as DEGs of flavonoid biosynthetic 
genes, but they could not determine regulatory genes of anthocyanin biosynthesis in $M$. incana flowers.

In the present study, we identified enzymatic and regulatory genes of anthocyanin biosynthesis in $M$. incana flowers, and we revealed genes corresponding to each locus reported previously. Moreover, we investigated the expression profiles of anthocyanin biosynthetic genes in the course of flower development in 'Vintage Lavender', which has native flower colors. We attempted to reveal the molecular regulatory mechanism underlying anthocyanin biosynthesis in $M$. incana flowers.

\section{Materials and methods}

\section{Plant materials}

The seeds of 'Vintage Lavender', which is a potted cultivar with purple and doubled flowers, were purchased from M\&B flora (Hokuto, Yamanashi, Japan). They were grown in a greenhouse located at Shizuoka University (Shizuoka, Japan) under natural conditions from September 2017 to April 2018. Petal samples were collected at four different flower developmental stages: S1, flower developmental stage 1, floral bud length of 5-10 mm, and petal not yet emerged from sepal; S2, floral bud length of 10-15 $\mathrm{mm}$ and pigmentation beginning at the top of petals; S3, flower length of 15-20 mm; and S4, flower length of $>20 \mathrm{~mm}$ and anthesis (Fig. 1a). All samples were frozen immediately using liquid nitrogen and stored at $-80^{\circ} \mathrm{C}$ until use.

Another seven different cultivars, i.e., 'Vintage Burgundy', 'Vintage Lilac', 'Vintage Red', 'Vintage Rose', 'Vintage Copper', 'Vintage Peach', and 'Vintage Yellow', were also grown under the same condition. For real-time quantitative PCR (RT-qPCR) and metabolic analyses, petal samples were collected at stages $\mathrm{S} 3$ and $\mathrm{S} 4$, respectively, and stored at $-80^{\circ} \mathrm{C}$ until use.

\section{Flavonoid analysis}

One gram of petal samples collected at different flower developmental stages as well as leaf and stem samples were ground to powder using liquid nitrogen and extracted with $5 \mathrm{~mL}$ of MAW (40\% methanol and $10 \%$ acetic acid) for $24 \mathrm{~h}$ at $4{ }^{\circ} \mathrm{C}$. Then, the extract was filtered by passage through a $0.22-\mu \mathrm{m}$ PTFE syringe filter (Osaka Chemical, Osaka,

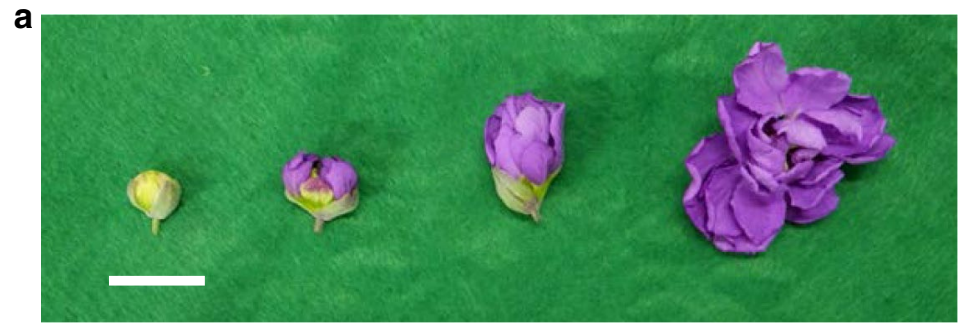

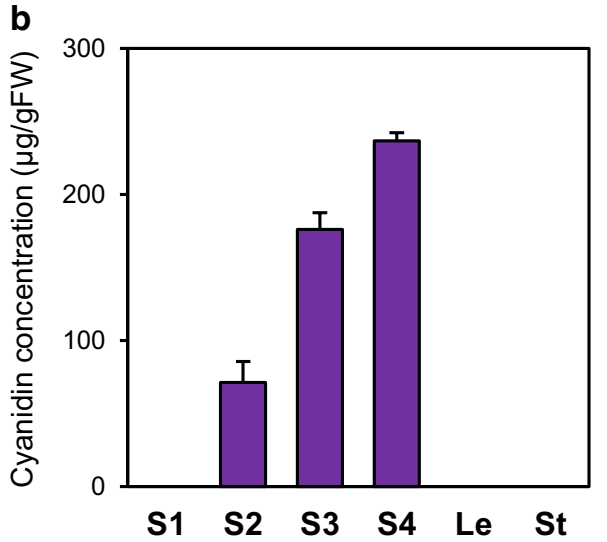

Fig. 1 Temporal and spatial accumulation patterns of flavonoids in Matthiola incana 'Vintage Lavender'. a Flower developmental stages (S1-S4) as described in the "Materials and methods" section. Bar $=1 \mathrm{~cm}$. b Anthocyanin accumulation in petals at four flower developmental stages (S1-S4), leaves (Le), and stems (St). Anthocyanins extracted from each sample were converted to aglycones through hydrolysis and quantified using cyanidin or pelargonidin as a stand-

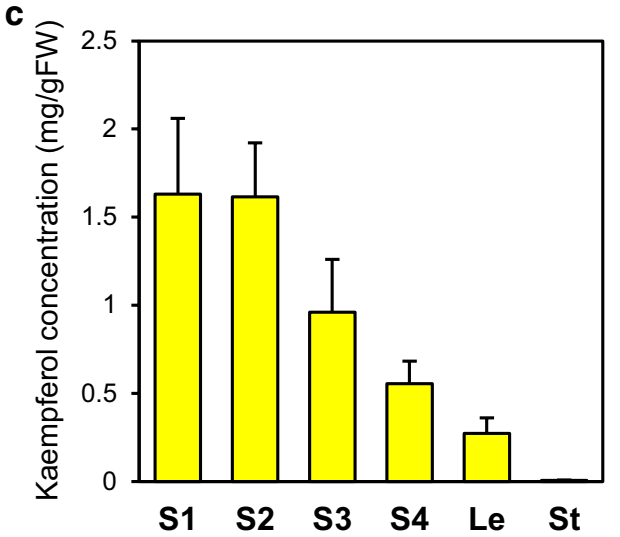

ard. Values are expressed as mean \pm standard error $(n=5)$. c Flavonol accumulation in petals at four flower developmental stages (S1-S4), leaves (Le), and stems (St). Flavonoids extracted from each sample were converted to aglycones by hydrolysis and quantified using kaempferol as a standard. Values are expressed as mean \pm standard error $(n=5)$ 
Japan). Anthocyanin and flavonol analyses were conducted using the Agilent Infinity 1290 High Performance Liquid Chromatography system (Agilent Technologies, Santa Clara, CA, USA), Agilent 1200 Diode-Array Detector SL (Agilent Technologies), and the reverse-phase column YMC-Pack Pro C18 RS (4.6×150 mm, $5 \mu \mathrm{m}$; YMC, Kyoto, Japan). Solvent A $[1.5 \%(\mathrm{v} / \mathrm{v})$ phosphoric acid] and solvent B $[1.5 \%$ (v/v) phosphoric acid containing $20 \%$ (v/v) acetic acid and $25 \%(\mathrm{v} / \mathrm{v})$ acetonitrile] were used for elution at $40{ }^{\circ} \mathrm{C}$ at a flow rate of $0.8 \mathrm{~mL} \mathrm{~min}{ }^{-1}$. Elution was performed with $20-85 \%$ of solvent B for 40 min under gradient elution conditions. Anthocyanidins and flavonols were monitored based on absorbance measured at wavelengths of 520 and $360 \mathrm{~nm}$, respectively.

To identify the types of anthocyanidins and flavonols accumulating in different organs, the extracted flavonoids were converted to aglycone by hydrolysis. Flavonoids were extracted using $5 \mathrm{~mL}$ of methanol with $1 \%$ hydrochloric acid for $24 \mathrm{~h}$ at $4{ }^{\circ} \mathrm{C}$. To convert flavonoids to aglycone by hydrolysis, $4.5 \mathrm{~mL}$ of $3 \mathrm{M}$ hydrochloric acid was added to $500 \mu \mathrm{L}$ of flavonoid extracts, and the mixture was boiled for $1 \mathrm{~h}$. Anthocyanidins extracted using isoamyl alcohol were dried and then resuspended in $500 \mu \mathrm{L}$ of methanol with $0.1 \%$ hydrochloric acid. On the other hand, flavonol aglycones extracted using ethyl acetate were dried and then resuspended in $500 \mu \mathrm{L}$ of $80 \%$ methanol. The flavonol extracts were filtered as mentioned above. Anthocyanidin and flavonol aglycone analyses were conducted using Agilent Infinity $1290 \mathrm{HPLC}$ with the reverse-phase column YMC-Triart C18 (2.0 $\times 50 \mathrm{~mm}, 1.9 \mu \mathrm{m}$; YMC, Kyoto, Japan), and they were used for elution at $40{ }^{\circ} \mathrm{C}$ at a flow rate of $1.0 \mathrm{~mL} \mathrm{~min}^{-1}$. Elution was performed with $10 \%$ solvent $\mathrm{A}$ for $1 \mathrm{~min}$ and then $60 \%$ solvent A for 8 min under gradient elution conditions. Anthocyanidins and flavonols were monitored based on absorbance measured at wavelengths of 530 and $360 \mathrm{~nm}$, respectively. Anthocyanidin and flavonol levels were determined using authentic standards (anthocyanidins: pelargonidin, cyanidin, and delphinidin; flavonols: kaempferol, quercetin, myricetin; Extrasynthese, Lyon, France). In flavonoid analyses, five biological replicates were used.

\section{RNA-seq of $M$. incana floral buds}

Total RNA was isolated from petal, leaf, and stem samples using RNAiso Plus (Takara Bio, Otsu, Shiga, Japan) and purified using lithium acetate precipitation. Total RNAs isolated from floral buds of 'Vintage Lavender' at stage S3 were treated using the DNA-free DNA Removal kit (Invitrogen, Thermo Fisher Scientific, Waltham, MA, USA) to eliminate genomic DNA contamination. RNA-seq analysis was performed by Eurofins Genomics (Tokyo, Japan) using 125base pair (bp) paired-ends on Illumina HiSeq2500. Adaptors and low-quality reads were discarded from raw sequences; then, data were assembled using Trinity v.2.4.0 (Grabherr et al. 2011) in DDBJ read annotation pipeline. The obtained contigs were defined as unigenes after the removal of any redundancy. The unigenes were submitted to A. thaliana protein database TAIR10 from The Arabidopsis Information Resource for homolog and annotation comparison using the BLASTX algorithm.

\section{Isolation of open reading frames (ORFs) of candidate genes}

To confirm the sequences of candidate anthocyanin biosynthetic genes in $M$. incana, we attempted to amplify their ORFs using 'Vintage Lavender' petal cDNA as a template. In brief, $25 \mu \mathrm{L}$ of PCR comprised $1 \times E x$ buffer, $0.2 \mathrm{mM}$ dNTPs, $0.4 \mu \mathrm{M}$ of each primer, $1.25 \mathrm{U}$ Ex Taq polymerase (Takara Bio), and $1 \mu \mathrm{L}$ of cDNA template. The primers used in the present study are listed in Supplementary Table S1. The reaction conditions were as follows: preheating at $94{ }^{\circ} \mathrm{C}$ for $2 \mathrm{~min} ; 35$ cycles at $95{ }^{\circ} \mathrm{C}$ for $20 \mathrm{~s}, 60{ }^{\circ} \mathrm{C}$ for $40 \mathrm{~s}$, and $72{ }^{\circ} \mathrm{C}$ for $2 \mathrm{~min}$; and then extension at $72{ }^{\circ} \mathrm{C}$ for $10 \mathrm{~min}$. The amplified fragments were subcloned into a pGEM-T Easy vector system (Promega, Madison, WI, USA). The sequences of all constructs were confirmed by DNA sequencing (Fasmac, Atsugi, Kanagawa, Japan).

\section{Gene expression analysis of anthocyanin biosynthetic genes}

To obtain the temporal and spatial expression profiles of anthocyanin biosynthetic genes in 'Vintage Lavender', total RNAs were isolated from petals of each floral organ at different developmental stages, leaves and stems as described above. To compare the gene expression levels of $\mathrm{MibHLH1}$, total RNA was isolated from petals of another seven different cultivars at stage S3. The elimination of genomic DNA and cDNA synthesis were performed using PrimeScript RT Reagent Kit with gDNA Eraser (Takara Bio). Real-time qPCR of each gene was performed using Thermal Cycler Dice Real Time System (TP850; Takara Bio) using the KAPA SYBR FAST qPCR Master Mix kit (KAPA Biosystems, Wilmington, MA, USA). Briefly, the reaction mixture $(10 \mu \mathrm{L})$ comprised $1 \times$ Master Mix, $0.2 \mu \mathrm{M}$ of each primer, and $1 \mu \mathrm{L}$ of template cDNA, and the cycling conditions were as follows: $95^{\circ} \mathrm{C}$ for $20 \mathrm{~s}$, followed by 40 cycles of $95^{\circ} \mathrm{C}$ for $1 \mathrm{~s}$ and $60{ }^{\circ} \mathrm{C}$ for $20 \mathrm{~s}$. The specificity of each amplification reaction was checked by the addition of a dissociation analysis step after the cycle reaction. Data were analyzed by second derivative maximum methods using Thermal Cycler Dice Real Time System II v5.0 (Takara Bio). The transcript level of each gene was calculated relative to that of $M$. incana actin-encoding MiACT2 (LC496063) as a reference gene. qPCR was performed using five biological replicates. The 
sequences of all primers used in qPCR are listed in Supplementary Table S1.

\section{Yeast two-hybrid analysis of anthocyanin biosynthetic transcription factors}

To investigate protein-to-protein interactions among MiMYB1, MibHLH1, MibHLH2, MiWDR1, and MiWDR2 proteins, we employed yeast two-hybrid analysis using Matchmaker Two-Hybrid System 3 (Clontech, Takara Bio). The coding regions of each transcription factor were cloned into either the pGAD-T7 (GAL4 activation domain) or pGBK-T7 (GAL4 DNA-binding domain) vector. All constructs were transformed into Saccharomyces cerevisiae AH109 (Clontech, Takara Bio). The transformed yeast cells were grown on SD selective medium without leucine ( $-\mathrm{Leu}$ ) and tryptophan (-Trp) at $30{ }^{\circ} \mathrm{C}$ for 3 days. The survival test for each transformed yeast culture was performed on selective quadruple dropout medium (- Leu, - Trp, histidine [-His], and adenine [-Ade]) supplemented with $15 \mathrm{mM}$ 3-amino-1,2,4-triazole (3-AT) at $30^{\circ} \mathrm{C}$ for 3 days.

\section{Turnip mosaic virus (TuMV) inoculation into $N$. benthamiana and $M$. incana seedlings}

pGTuMVUK1 is a binary vector constructed from full length TuMV UK1 isolate under the control of CaMV35S promoter (Sánchez et al. 1998; Llorente et al. 2019). pBTuMVUK1DAE-EZE is an intermediate cloning vector partly containing nucleotide sequences of both nuclear inclusion $(\mathrm{NIb})$ and coat protein $(\mathrm{CP})$ from TuMV UK1 isolate. Green fluorescent protein $(G F P)$ and MiMYBl ORF without stop codon were inserted into pBTuMVUK1DAE-EZE digested by Esp 3 I using NEBuilder HiFi assembly master mix (New England BioLabs Japan, Tokyo, Japan), and the constructed plasmids were designated as pBTuMVUK1:GFP and pBTuMVUK1:MiMYB1, respectively. pBTuMVUK1:GFP and pBTuMVUK1:MiMYB1 were double-digested using Mlu I and Apa I, and then the digested fragments were ligated with pGTuMVUK1 treated with the same restriction enzymes. They were designated as pGTuMVUK1:GFP and pGTuMVUK1:MiMYB1 (Fig. 4a). Agrobacterium tumefaciens GV3101 harboring pCLEAN-S48, which is the helper plasmid for pGreen (Thole et al. 2007), was transformed with pGTuMVUK1:GFP or pGTuMVUK1:MiMYB1, and selected on YEB solid medium supplemented with $30 \mathrm{mg} \mathrm{L}^{-1}$ kanamycin, $7.5 \mathrm{mg} \mathrm{L}^{-1}$ tetracycline, and $25 \mathrm{mg} \mathrm{L}^{-1}$ rifampicin, at $28{ }^{\circ} \mathrm{C}$ for 3 days. A single colony was cultured overnight in YEB liquid medium containing $30 \mathrm{mg} \mathrm{L}^{-1}$ kanamycin and $7.5 \mathrm{mg} \mathrm{L}^{-1}$ tetracycline at $28^{\circ} \mathrm{C}$. A. tumefaciens cells were harvested and suspended in infiltration buffer $\left(10 \mathrm{mM} \mathrm{MgCl}_{2}, 200 \mathrm{mM}\right.$ acetosyringone, and $10 \mathrm{mM}$ MES, pH 5.6), and the suspensions were adjusted to an optical density of 1.0 at $600 \mathrm{~nm}\left(\mathrm{OD}_{600}\right)$. A. tumefaciens suspension was infiltrated into Nicotiana benthamiana leaves using a needleless $1 \mathrm{~mL}$ syringe (Terumo, Tokyo, Japan). At 7 day after Agrobacterium infiltration, upper symptomatic leaves of $N$. benthamiana infected with each virus construct were used as inoculum for 1-monthold seedlings of $M$. incana 'Kiss me Violet' (Takii-seed, Kyoto, Japan). Crude $N$. benthamiana infectious extract was prepared by grinding the leaves with an infiltration buffer at a four-fold volume relative to leaf fresh weight. The crude sap was rub-inoculated onto mature leaves dusted with carborundum. Inoculated garden stock seedlings were grown at $25{ }^{\circ} \mathrm{C}$ under 16 photoperiod of fluorescent lamp.

Anthocyanins were extracted from 100-mg leaves of TuMV-inoculated plants using $1 \mathrm{~mL}$ of $80 \%$ methanol with $0.1 \%$ hydrochloric acid, and they were measured using HPLC as described above. Anthocyanin concentration was calculated on the basis of a standard curve of cyanidin-3-glucoside. The expression levels of transgene and endogenous anthocyanin biosynthetic genes of TuMV:GFP-inoculated or TuMV:MiMYB1-inoculated plants were investigated using RT-qPCR as described above. As an internal reference gene in RT-qPCR for $N$. benthamiana plants, we used ubiquitin $C$ (AB026056.1, Yang et al. 2019).

\section{Results}

\section{Flavonoid profiles of 'Vintage Lavender'}

The extract derived from the petals of 'Vintage Lavender' showed two major peaks at $520 \mathrm{~nm}$ on chromatograph, which were identified as cyanidin 3-[2-(2-(sinapoyl)-xylosyl)-6-( $p$ coumaroyl)-glucoside]-5-[6-(malonyl)-glucoside] and cyanidin 3-[2-(2-(sinapoyl)-xylosyl)-6-(feruloyl)-glucoside]-5-[6(malonyl)-glucoside] (Supplementary Fig. S1a; Tatsuzawa et al. 2012). On the other hand, three major peaks detected at $360 \mathrm{~nm}$ were identified as 1-(sinapoyl)-glucopyranoside, kaempferol 3-(2-rhamnopyranosyl)- $\beta$-glucopyranoside-7rhamnopyranosid, and kaempferol 3-(2-rhamnopyranosyl)arabinopyranoside-7-rhamnopyranoside (Supplementary Fig. S1b; Tatsuzawa et al. 2014). After hydrolyzed flavonoids were extracted from 'Vintage Lavender' petal, cyanidin and kaempferol were detected as aglycones (Supplementary Fig. S1c-f; Tatsuzawa et al. 2014). Therefore, cyanidin and kaempferol derivatives were thought to be responsible for purple flower color of 'Vintage Lavender'.

To investigate the temporal and spatial accumulation profiles of anthocyanins and flavonols, we hydrolyzed flavonoid extracts and then determined cyanidin and kaempferol levels. The accumulation levels of cyanidin derivatives in petals increased from stage S2 and then peaked [263 $\mu \mathrm{g} \mathrm{g}^{-1}$ fresh weight (FM)] at stage $\mathrm{S} 4$, which is the anthesis stage 

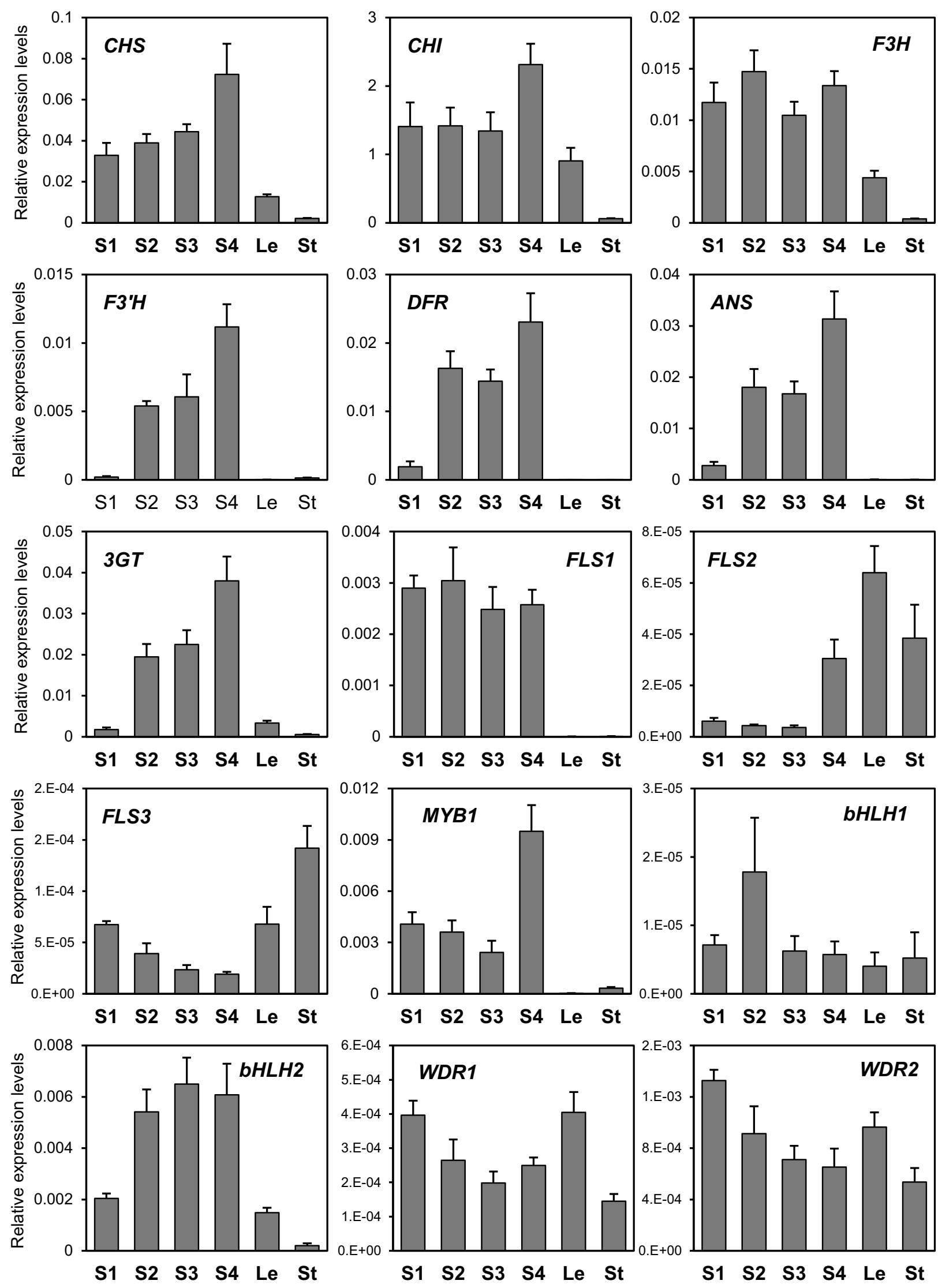
4Fig. 2 Relative expression levels of anthocyanin biosynthetic genes in petals, leaves, and stems of Matthiola incana 'Vintage Lavender'. The expression levels of chalcone synthase $(\mathrm{MiCHS})$, chalcone isomerase $(\mathrm{MiCHI})$, flavanone 3-hydroxylase $(\mathrm{MiF} 3 \mathrm{H})$, flavonoid 3'-hydroxylase $\left(M i F 3^{\prime} H\right)$, dihydroflavonol 4-reductase $(M i D F R)$, anthocyanidin synthase $(M i A N S)$, anthocyanidin 3-O-glucosyltransferase (Mi3GT), flavonol synthase 1 (MiFLS1), MiFLS2, and MiFLS3, MiMYB1, Mib$H L H 1, M i b H L H 2, M i W D R 1$, and MiWDR2 were normalized using that of MiACT2. Stages S1-S4 indicate petal samples at flower developmental stages S1-S4. Le and St indicate leaf and stem samples, respectively. Values are expressed as mean \pm standard error $(n=5)$

(Fig. 1b). However, no anthocyanin accumulation was detected in their leaves and stems. In contrast, kaempferol derivatives as flavonols were detected in the petals, leaves, and stems of 'Vintage Lavender' (Fig. 1c). The highest kaempferol level $\left(1,631 \mu \mathrm{g} \mathrm{g}^{-1} \mathrm{FW}\right)$ was detected in petals at the early developmental stages (stages S1 and S2), which then reduced with petal development. In leaves, $273 \mu \mathrm{g} \mathrm{g}^{-1}$ FW of kaempferol was accumulated, whereas $5.5 \mu \mathrm{g} \mathrm{g}^{-1} \mathrm{FW}$ of kaempferol accumulation was detected in stems. Consequently, the accumulation profile of flavonols was different from that of anthocyanins in M. incana.

\section{Identification of anthocyanin biosynthetic genes using NGS}

To construct a unigene catalog of 'Vintage Lavender' flowers, we performed RNA-seq analysis on floral buds at stage S3 using 125-bp paired-end reads on Illumina Hiseq2500. After quality filtering and read cleaning, we obtained approximately 16 million raw sequencing reads, from which 130,224 contigs were assembled with an average sequence length of 1,508 bp. In addition, 73,286 contigs assembled from 32 million raw sequencing reads (SRR5195384 and SRR5195385) deposited by Chen et al. (2018) were used to identify orthologs. Based on a BLASTX search using the amino acid sequences of anthocyanin biosynthetic enzymatic genes in $A$. thaliana as queries, single orthologs for $C H S, C H I, F 3 H, F 3^{\prime} H, D F R, A N S$, and $3 G T$ were obtained from $M$. incana contigs. However, we could not find $F 3^{\prime} 5^{\prime} H$ orthologs from these floral contigs, suggesting that $M$. incana does not synthesize any delphinidin derivatives. The deduced amino acid sequences of $M$. incana orthologs exhibited 82.5-95.0\% identities with those of corresponding proteins (Supplementary Table S2). On the other hand, three $F L S$ orthologs were obtained from $M$. incana contigs, which were termed MiFLS1, MiFLS2, and MiFLS3. The deduced amino acid sequences of MiFLS1, MiFLS2, and MiFLS3 exhibited $60.7 \%, 64.0 \%$, and $52.7 \%$ identities, respectively, with those of A. thaliana FLS1. The deduced amino acid sequence of MiFLS1 exhibited 57.0\% and $47.5 \%$ identities with those of MiFLS2 and MiFLS3, respectively.
Transcription factor genes associated with anthocyanin biosynthesis were obtained from $M$. incana contigs, particularly one $M Y B$ (MiMYB1), two bHLH (MibHLH1 and MibHLH2), and two WDR orthologs (MiWDRl and MiWDR2). The deduced amino acid sequence of MiMYB1 exhibited $72.2 \%$ and $67.6 \%$ identities with those of $B$. oleracea var. botrytis $M Y B 2$ and $A$. thaliana $P A P 1$, respectively (Supplementary Fig. S2 and Table S2). MibHLH1 and MibHLH2 were classified into different clades belonging to the TT8 and EGL3 families, respectively (Supplementary Fig. S3 and Table S2). The deduced amino acid sequence of $\mathrm{Mib}$ $H L H 1$ exhibited $78.4 \%$ and $72.5 \%$ identities with those of $A$. thaliana TT8 and B. oleracea var. botrytis TT8 (ADP76654), respectively. On the other hand, the deduced amino acid sequences of MibHLH2 exhibited $84.6 \%$ and $80.0 \%$ identities with those of $A$. thaliana EGL3 and $B$. rapa subsp. rapa EGL3 (ADK11703), respectively. MibHLH1 exhibited $31.3 \%$ identity with MibHLH2 in terms of amino acid sequences.

MiWDR1 and MiWDR2 were classified into different clades, including TTG1 and AN11 families, respectively (Supplementary Fig. S4 and Table S2). Their partial sequences have been deposited in GenBank (accession numbers CAE53274 and CAE76645, respectively; Dressel and Hemleben 2009). The deduced amino acid sequence of Mib$W D R 1$ exhibited $96.5 \%$ and $93.2 \%$ identities with those of $A$. thaliana TTG1 and B. oleracea var. botrytis TTG1, respectively. The deduced amino acid sequence of MibWDR2 displayed $99.1 \%$ identity with that of A. thaliana AN11, whereas MiWDR1 exhibited 62.6\%identity with MiWDR2.

\section{Temporal and spatial expression profiles of flavonoid biosynthetic genes}

To investigate temporal and spatial expression profiles of candidate anthocyanin biosynthetic genes, we performed RT-qPCR (Fig. 2). High expression levels of $\mathrm{MiCHS}$, $\mathrm{MiCHI}$, and $\mathrm{MiF3H}$ were detected in petals at all floral developmental stages and in leaves. The expression levels of $M i F 3^{\prime} H, M i D F R, M i A N S$, and Mi3GT increased markedly at stage $\mathrm{S} 2$ and then peaked at stage $\mathrm{S} 4$. However, no or slight expression level of these genes was detected in leaves and stems. Therefore, the temporal and spatial profiles of $M i F 3 ' H, M i D F R, M i A N S$, and Mi3GT transcripts were correlated well with those of anthocyanin accumulation (Figs. 1b and 2).

Among the three MiFLS genes, MiFLS1 was expressed at high levels in petals compared with MiFLS2 and MiFLS3 (Fig. 2). MiFLS1 constantly exhibited high expression levels in the course of petal development; however, it was not observed in leaves and stems. However, the expression levels of MiFLS2 and MiFLS3 were higher in leaves and stems than in petals. 
We also investigated the temporal and spatial expression profiles of five regulatory genes involved in anthocyanin biosynthesis. MiMYBI expression in petals decreased from stages $\mathrm{S} 1$ to $\mathrm{S} 3$ and then increased remarkably at stage S4 (Fig. 2). MiMYBl was expressed below detectable levels in leaves and stems. In addition, MibHLHI expression was the highest at stage S2, but it was considerably lower than those of other regulatory genes of anthocyanin biosynthesis in the petals of 'Vintage Lavender'. In contrast, MibHLH2 expression increased with flower development and was detected in leaves. Both MiWDR 1 and MiWDR2 were expressed constantly in petals at different flower developmental stages as well as in leaves and stems (Fig. 2). Therefore, the MibHLH2 expression profile was highly correlated with anthocyanin accumulation profile in M. incana (Figs. 1b and 2).

\section{Protein-to-protein interactions among MiMYB1, MibHLHs, and MiWDRs}

To confirm protein-to-protein interaction among the five transcription factors of $M$. incana, we employed the GAL4 yeast two-hybrid system (Fig. 3). The yeasts harboring MiMYB1-MibHLH1, MiMYB1-MibHLH2, MiMYB1-MiWDR1, MibHLH1-MiWDR1, and MibHLH2-MiWDR1 combinations grew on quadruple dropout medium, indicating protein-to-protein interactions (Fig. 3). However, the MibMYB1-MiWDR1 combination grew only on yeasts harboring AD:MYB1 and BK:MiWDR1 on quadruple dropout medium. On the other hand, MiWDR2 could not form homodimers or heterodimers with any other transcription factor. Taken together, these results suggest that
MiMYB1 can form heterodimers with MibHLH1 or MibHLH2 and that they can form either trimeric or tetrameric complex with MiWDR1.

\section{Ectopic expression of MiMYB1 using TuMV in seedlings of $N$. benthamiana and $M$. incana}

To investigate whether MiMYB1 actually plays the role of a regulatory gene in anthocyanin biosynthesis, we performed a transient expression analysis of this transcription factor using a TuMV vector in $M$. incana leaves. High expression levels of other transcription factor genes, including $M i b$ HLH1, MibHLH2, MiWDR1, and MiWDR2, were measured in leaves, but no expression level of MiMYB1 was detected in leaves compared with those in petals (Fig. 2). Therefore, we attempted to transiently express MiMYB1 in mature leaves of 'Kiss me Violet' seedlings. 'Kiss me Violet' is a cultivar with glabrous leaves, which has a higher efficiency for sap inoculation from $N$. benthamiana than 'Vintage Lavender' with haired leaves.

To produce virus particles, A. tumefaciens GV3101 (containing the helper plasmid pCLEAN-S48) harboring pGTuMVUK1:MiMYB1 or pGTuMVUK1:GFP was infiltrated into $N$. benthamiana leaves. Six days after inoculation, RT-qPCR showed TuMV proliferation and transgene (MiMYBI and GFP) expression in the leaves inoculated with TuMVUK1:MiMYB1 or TuMVUK1:GFP (Supplementary Fig. S5a). Anthocyanin accumulation in the form of red pigmentation was observed in TuMVUK1:MiMYB1infected leaves (Supplementary Fig. S5b and c). On the other hand, TuMVUK1:GFP-infected leaves displayed GFP
Fig. 3 Protein-to-protein interactions among MiMYB1, MibHLHs, and MiWDRs. MiMYB1, MibHLH1, MibHLH2, MiWDR1, and MiWDR2 proteins were fused to the GAL4 DNA-binding domain or GAL4 activation domain. pGBKT7 and pGADT7 were used as the negative controls for bait and prey, respectively. Yeast was grown on double-selection medium (- Leu, and - Trp; right) and quadruple dropout medium (- Leu, - Trp, - His, and - Ade) supplemented with $15 \mathrm{mM}$ 3-AT (left) at $30{ }^{\circ} \mathrm{C}$ for 2-3 days

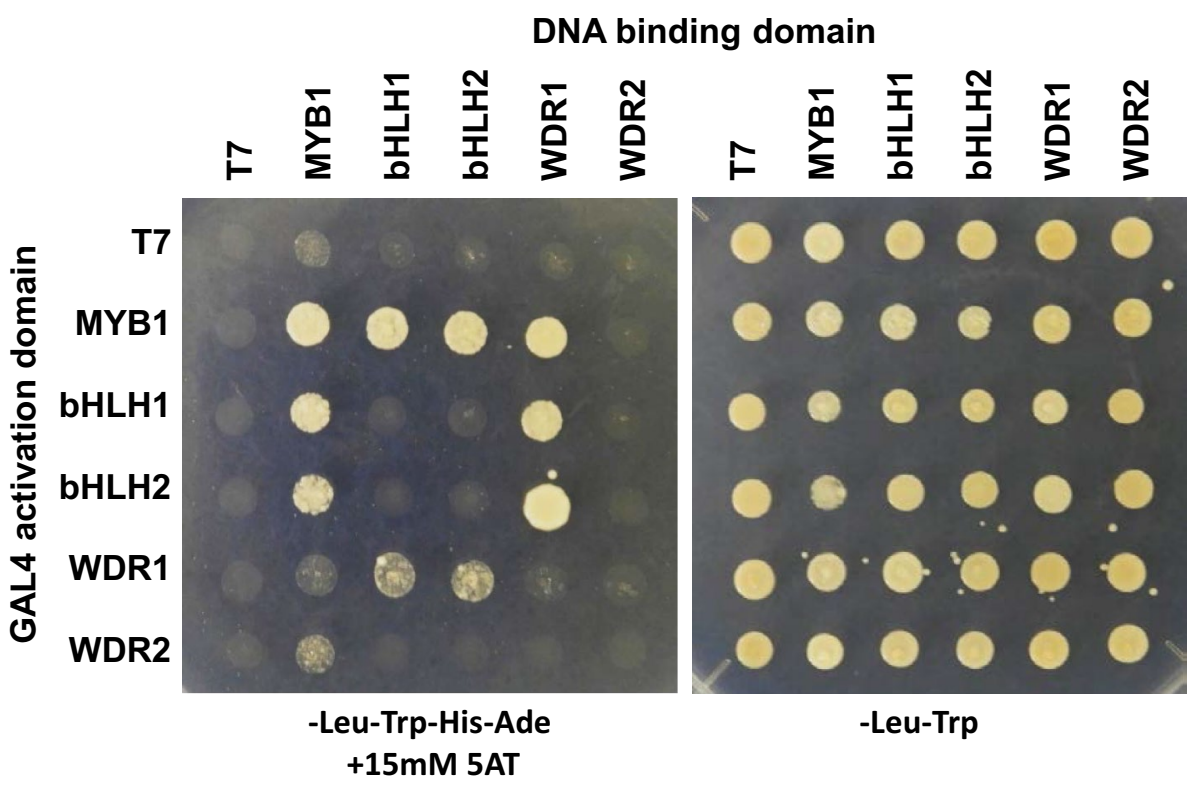


fluorescence but not anthocyanin pigmentation (Supplementary Fig. S5b, d, and e).

Extracts derived from TuMVUK1:MiMYB1-infected or TuMVUK1:GFP-infected $N$. benthamiana leaves were used to inoculate 1-month-old 'Kiss me Violet' seedlings. Seven days after inoculation, ectopic anthocyanin accumulation was initiated in the upper leaves of TuMVUK1:MiMYB1-infected plants; this accumulation increased up to 1 month after inoculation (Fig. 4b, Supplementary Fig. S6). On the other hand, no pigmentation was observed in any leaves of the control TuMVUK1:GFPinfected plants (Fig. 4b, Supplementary Fig. S6). RTqPCR revealed the propagation of recombinant TuMV to the upper leaves of independent infected plants (Fig. 4c). The upper leaves of TuMVUK1:MiMYB1-infected plants showed 5500-fold higher MiMYB1 expression levels than those of TuMVUK1:GFP-infected plants. In addition, the expression levels of endogenous anthocyanin biosynthetic genes, i.e., MiCHS, MiF3'H, MiDFR, and MiANS, enhanced by $6.6,137,2059$, and 3.8 folds, respectively, in TuMVUK1:MiMYB-infected plants (Fig. 4c). However, no difference in expression levels was observed for other regulatory genes of anthocyanin biosynthesis, i.e., MibHLH2 and MiWDRl, and flavonol biosynthesis, i.e., MiFLS1, between TuMVUK1:MiMYB1-infected and TuMVUK1:GFPinfected plants. These results strongly suggested that MiMYB1 activates the transcription of anthocyanin biosynthetic genes and induces anthocyanin accumulation in $M$. incana.

\section{Expression of MibHLH1 among different cultivars with colored flowers}

To determine the function of MibHLH1, we also investigated anthocyanin accumulation and MibHLHI expression using eight cultivars of 'Vintage series' with different flower colors. In the petals of 'Vintage Lavender' and 'Vintage Burgundy', the accumulation of cyanidin derivatives as anthocyanins was detected, whereas in other cultivars, only pelargonidin derivatives were detected (Fig. 5a). Among the eight cultivars, 'Vintage Burgundy' had the highest anthocyanin accumulation $\left(1,662 \mu \mathrm{g} \mathrm{g}^{-1} \mathrm{FW}\right)$, which was 6.3 -fold higher than that detected in 'Vintage Lavender' (Fig. 5a). The petals of 'Vintage Red', 'Vintage Rose', and 'Vintage Copper' also had higher accumulation levels of pelargonidin derivatives $\left(917,463\right.$, and $301 \mathrm{\mu g} \mathrm{g}^{-1} \mathrm{FW}$, respectively) than those of 'Vintage Lavender'. Thus, different accumulation levels of anthocyanin pigments were observed among the eight cultivars.

We compared the expression levels of MibHLH1 among the eight cultivars using the petals at stage $\mathrm{S} 3$, at which we detected strong expression levels of almost all anthocyanin biosynthetic genes in 'Vintage Lavender' (Fig. 2). Higher expression levels of $\mathrm{MibHLH1}$ were detected in 'Vintage Burgundy', 'Vintage Red', 'Vintage Rose', and 'Vintage Copper' than in 'Vintage Lavender' (Fig. 5b). The weak expression levels of MibHLH1 in the petals of other cultivars were similar to that observed in 'Vintage Lavender'. Thus, the expression levels of MibHLHI correlated well with anthocyanin accumulation levels depending on each cultivar of 'Vintage series' (Fig. 5a, b). It is possible that MibHLH1 plays an important role in controlling the amount of anthocyanin accumulation in $M$. incana petals.

\section{Discussion}

$M$. incana was one of the classic materials for genetic study in the early twentieth century, and it was used to identify loci related to flower pigments, flower shape, and leaf color (Forkmann 1993; Saunders 1928). However, only few recent studies have investigated anthocyanin biosynthetic genes in M. incana (Dressel and Hemleben 2009; Hemleben et al. 2004; Chen et al. 2018). Here, we identified anthocyanin biosynthetic genes associated with flower coloration in $M$. incana using NGS. The construction of cDNA libraries and use of NGS-based technologies are critical for performing functional analyses, particularly on horticultural plants lacking genome information. NGS-based technologies have been used to identify numerous genes associated with key floral traits in Chrysanthemum morifolium (Sasaki et al. 2017), Lilium hybrid (Suzuki et al. 2016), Paeonia lactiflora (Zhao et al. 2014), and Cymbidium hybrid (Nakatsuka et al. 2019). We obtained a total of 130,224 contigs with an average sequence length of 1,508 bp from the floral organs of 'Vintage Lavender'. Chen et al. (2018) reported 52,789 transcripts of $M$. incana and DEGs between five colored petals including white, light purple, rose bengal, dark purple, and pink petals. Twelve anthocyanin biosynthetic genes, including CHS, CHI, F3H, ANS, and UGT78D2, have been identified in each comparative analysis of DEG. Nevertheless, anthocyanin regulatory genes involved in the MBW complex, except MiTTG1 (Dressel and Hemleben, 2009), have not been identified yet.

$M$. incana belongs to Brassicaceae, which contains the model plant $A$. thaliana and important vegetative crops (e.g., cabbage, cauliflower, kale, and radish) (Dole and Wilkins 2005). Using known sequences of anthocyanin biosynthetic genes from $A$. thaliana as queries, we found their orthologs in the $M$. incana floral contigs constructed in the present study. The deduced amino acid sequences of $C H S, C H I$, $F 3 H, F 3^{\prime} H, D F R, A N S$, and $3 G T$ in $M$. incana displayed 82.5-95.0\% identities with those of the corresponding proteins in A. thaliana (Supplementary Table S2). Although we also attempted to identify regulatory genes of anthocyanin biosynthesis from the $M$. incana cDNA library, similarities 
a pGTUMVUK1:GFP

\begin{tabular}{|l|l|l|l|l|l|l|l|l|l|}
\hline 35Sp P1 & HC-Pro & P3 & C1 & VPg & Pro & NIb & GFP & CP \\
\hline
\end{tabular}

pGTUMVUK1:MiMYB1

\begin{tabular}{|l|l|l|l|l|l|l|l|l|l|}
\hline 35Sp P1 & HC-Pro & P3 & C1 & VPg & Pro & NIb & MYB1 & CP & hos T \\
\hline
\end{tabular}

b

TuMV:GFP

TuMV:MiMYB1
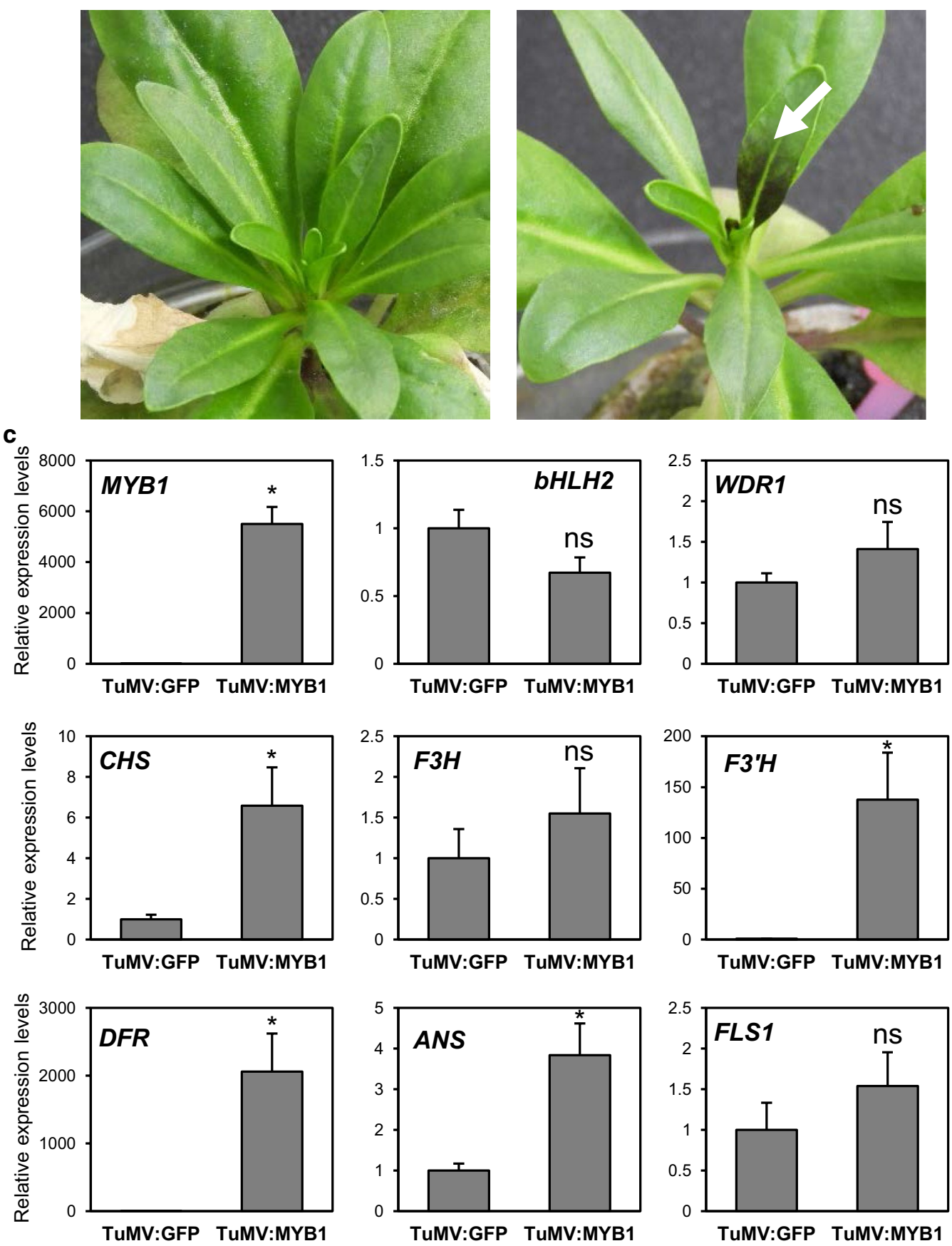
४Fig. 4 Ectopic expression of MiMYB1 in Matthiola incana seedling. a pGTuMVUK1:GFP and pGTuMVUK1:MiMYB1 vectors. b TuMVUK1:GFP-infected seedling (left), TuMVUK1:MiMYB1infected seedling (right) at 28 days after inoculation. The upper leaf that induced anthocyanin accumulation is showed by an arrow. c RT-qPCR of anthocyanin biosynthetic genes in TuMV-infected plants. Total RNA was isolated from the first leaf above the inoculated portion at 6 days after inoculation. TuMVUK1:GFP and TuMVUK1:MiMYB1 infection were performed to three and five independent 1-month-old seedlings, respectively, of 'Kiss me Violet'. Values are expressed as mean \pm standard error $(n=3-5)$. Asterisks indicate significant difference between TuMV:GFP and TuMV:MYB1 using Student's $t$-test $(P<0.05)$. ns indicates no significant difference

in amino acid sequences of regulatory genes of anthocyanin biosynthesis were lower than those of anthocyanin structural genes (Supplementary Table S2). Therefore, we performed more functional analyses to characterize these genes. In plants, the regulation of anthocyanin biosynthesis is controlled by the MBW complex, which is composed of three proteins, namely, R2R3-MYB, bHLH, and WDR (Xu et al. 2015). In A. thaliana, $P A P 1 / 2$ and TT2 have been reported to be anthocyanin and proanthocyanidin biosynthetic $R 2 R 3$ $M Y B$ genes, respectively (Bernhardt et al. 2003; Nesi et al. 2001). Only MiMYB1 was found in M. incana floral contigs and was categorized into a clade together with PAP1/2 (Supplementary Fig. S1). However, a TT2 ortholog was not found within the $M$. incana floral contigs constructed in the present study. A. thaliana TT2 and B. napus BnTT2 are expressed in seeds and regulate proanthocyanidin biosynthesis (Nesi et al. 2001; Wei et al. 2007). M. incana TT2 ortholog may also be expressed only in seeds, which may have precluded identification in the present study. We identified two bHLH transcription factors, i.e., MibHLH1 and MibHLH2, from $M$. incana floral contigs. According to phylogenetic tree analysis, MibHLH1 and MibHLH2 were classified into TT8 and EGL3 clades, respectively (Supplementary Fig. S3). In A. thaliana, TT8 and EGL3 participate in proanthocyanidin biosynthesis in seed coats and anthocyanins in leaves, respectively (Bernhardt et al. 2003; Nesi et al. 2001; Zhang et al. 2003). MibHLH2 expression increased with petal development, and it was highly correlated with the anthocyanin accumulation profiles (Figs. $1 \mathrm{~b}$ and 2). On the other hand, MibHLH1 is an ortholog of A. thaliana TT8, and weak MibHLH1 expression levels were observed in the petals of 'Vintage Lavender' (Fig. 2). However, cultivars with abundant anthocyanin accumulation such as 'Vintage Burgundy', 'Vintage Red', 'Vintage Rose', and 'Vintage Copper' had higher MibHLH1 expression levels than 'Vintage Lavender' (Fig. 5). In 'Vintage burgundy', the temporal expression of MibHLH1 in petals increased with flower development, and it was similar to that of MibHLH2 (data not shown). Ramsay et al. (2003) also reported that the transient expression of two A. thaliana bHLH genes, i.e., GL3 and MYC146 (=EGL3), in petals of a white-flowered $M$. incana mutant were associated with a lack of anthocyanin. Consequently, MibHLH2, which belongs to the GL3/EGL3 clade, might play a key role as a component of the MBW complex with MiMYBI and MiWDRl in anthocyanin biosynthesis in $M$. incana. Moreover, MibHLH1 might enhance anthocyanin accumulation when it coexists with the MBW complex. The nucleotide sequences of $M i W D R 1$ and MiWDR2 exhibited high similarity with TTG1 and TTG1-like (AN11) genes, respectively, as reported previously (Dressel and Hemleben 2009).

Based on the expression profiles of anthocyanin biosynthetic genes in 'Vintage Lavender', they could be classified into three groups. Group 1 included $M i F 3 ' H, M i D F R$, $M i A N S$, and Mi3GT, and temporal expression levels of these genes increased with petal development (Fig. 2). The expression profiles of genes belonging to group 1 were highly correlated with the anthocyanin accumulation profiles (Figs. 1b and 2). Group 2 included MiFLS1, which exhibited constant expression levels in petals throughout flower development (Fig. 2). In addition, the expression profiles of genes belonging to group 2 tended to resemble flavonol accumulation profiles (Figs. 1c and 2). Finally, group 3 contained $\mathrm{MiCHS}$, $\mathrm{MiCHI}$, and $\mathrm{MiF3H}$, whose temporal expression profiles seem to be consistent with those of combined groups 1 and 2. The expression of group 3 genes was observed not only in petals but also in leaves (Fig. 2). MiCHS, MiCHI, and $\mathrm{MiF3H}$ were essential enzymes for anthocyanin and flavonol biosynthesis. In petals, flavonol derivatives were predominantly produced during the early floral developmental stage (S1); then, the biosynthesis of anthocyanin derivatives increased according to the transcription activation of group 1 genes during the late floral developmental stages (S2-S4). The targeted genes of the anthocyanin biosynthetic pathway under the control of a regulatory gene have been reported to be conserved in plant species such as petunia (Quattrocchio et al. 1993) and Japanese gentian (Nakatsuka et al. 2005, 2008).

Yeast two-hybrid analysis revealed that MiMYB1 formed a heterodimer with MibHLH1 and MibHLH2 (Fig. 3). In addition, MiWDR1 formed a heterodimer with MiMYB1, MibHLH1, and MibHLH2 (Fig. 4). These protein-to-protein interaction data support that the fact that trimeric complexes MiMYB1-MibHLH1-MiWDR1 or MiMYB1-MibHLH2-MiWDR1 could form and regulate anthocyanin biosynthesis. Although a constant level of $M i W D R 1$ expression was observed in the whole plant, the MiMYB1 and MibHLH2 expression levels in petals were higher than those in leaves and stems (Fig. 2). Therefore, the coexpressions of MiMYB1 and MibHLH2 in petals would activate the transcriptions of group 1 enzymatic genes including $M i D F R, M i A N S$, and $M i 3 G T$, resulting in enhanced anthocyanin accumulation with flower development. 

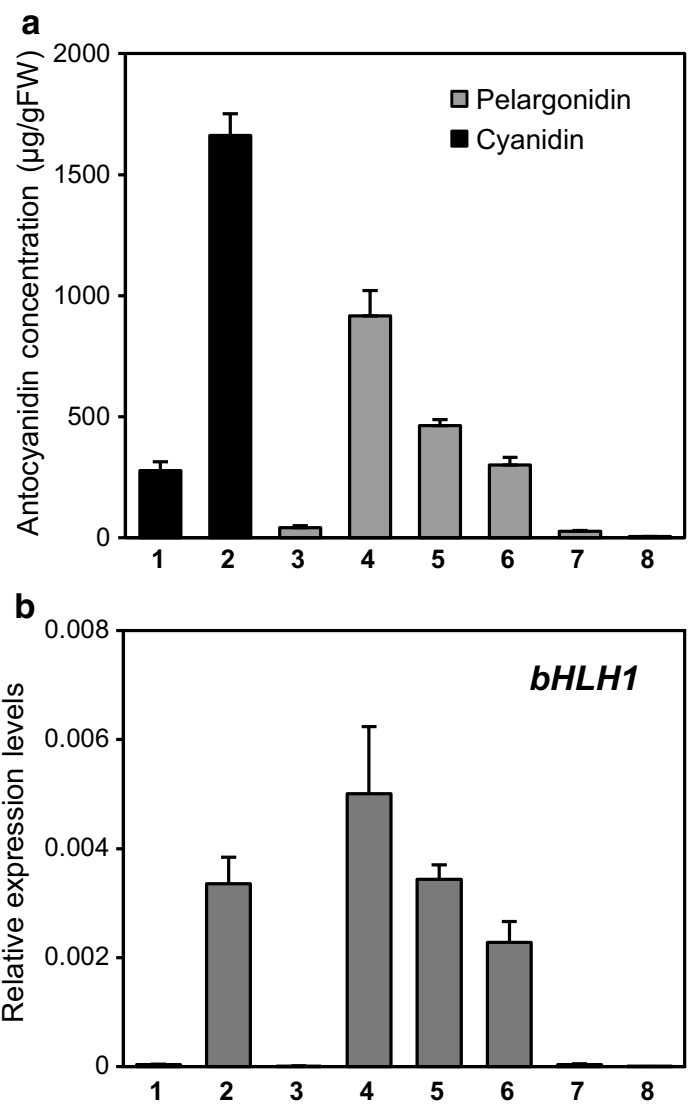

Fig. 5 Anthocyanin accumulation and expression level of MibHLH1 in eight different cultivars with colored flowers of Matthiola incana. a Anthocyanidin accumulation levels in petals of each cultivar at stage S4. b Relative expression levels of MibHLH1 in petals of each cultivar at stage S3. 1, 'Vintage Lavender'; 2, 'Vintage Burgundy'; 3, 'Vintage Lilac'; 4, 'Vintage Red'; 5, 'Vintage Rose'; 6, 'Vintage Copper'; 7, 'Vintage Peach'; 8, 'Vintage Yellow'. Values are expressed as mean \pm standard error $(n=5)$

To investigate whether MiMYBI can activate anthocyanin biosynthesis, we developed a transient expression system using a TuMV vector in M. incana (Fig. 4). It is recalcitrant to develop stable transgenic plants in $M$. incana for the investigation of gene function, and no transformant has been reported yet. As an alternative to stable transformants, the overexpression or suppression of genes using virus vectors is a useful tool for investigating gene function (Mortimer et al. 2015). Tobacco rattle virus (TRV, Tian et al. 2014) and Apple latent spherical virus (ALSV, Kamada et al. 2018) vectors have been used for the overexpression of foreign genes in several horticultural plants. We attempted to transiently overexpress foreign genes in $M$. incana using TRV and ALSV vectors, but no reproducible results were obtained (data not shown). In contrast, the transient expression of MiMYBl using
TuMV induced anthocyanin accumulation in infected leaves 6 days after infection (Fig. 4b). The upper leaves of TuMVUK1:MiMYB1-infected $M$. incana plants had 5,500-fold higher expression levels of MiMYB1 than those of TuMVUK1:GFP-infected plants (Fig. 4c). Similarly, the expression levels of endogenous $M i C H S, M i F 3^{\prime} H$. MiDFR, and MiANS in TuMVUK1:MiMYB1-infected plants were significantly higher than those in control plants. However, the expression levels of MiCHI, MiF3H, Mi3GT, MiFLSI, MibHLH1, MiWDR1, and MiWDR2 were not activated by the ectopic expression of MiMYB1 in leaves (Fig. 4c and data not shown). High expression levels of MibHLH2 and $M i W D R 1$, which are other regulatory factors of the MBW complex, were observed in petals and leaves of $M$. incana (Fig. 2). These results indicated that MiMYB1 was a key gene strictly regulating anthocyanin biosynthesis in the petals of M. incana and that the MiMYB1-MibHLH2-MiWDR 1 complex activates the transcription of endogenous enzymatic genes such as MiF3'H, MiDFR, and MiANS (Fig. 6). Antirrhinum majus ROSEAl (Rosl), an anthocyanin-related R2R3-MYB transcription factor, has been used for the visual tracking of plant viral infection and movement (Bedoya et al. 2012). Tobacco etch virus:Ros1-inoculated tobacco plants and TuMV:Ros1inoculated $A$. thaliana plants turned purple and had induced anthocyanin accumulation (Bedoya et al. 2012). In the present study on $M$. incana, TuMVUK1:MiMYB1 could also track plant viral infection and movement (Fig. 4b). Therefore, the transient expression system developed in the present study using a TuMV vector could facilitate not only the functional analysis of candidate genes derived from $M$. incana but also the characterization of TuMV.

In radish, the activation of $R S D F R$ expression was controlled by $R s M Y B 1$ and enhanced by the presence of RsTT8 (Lim et al. 2017). In red cabbage, the transcriptional upregulation of BoMYB2 and BoTT8 appears to be essential for the activation of anthocyanin structural genes for anthocyanin production during different developmental stages of vegetative growth (Yuan et al. 2009). In $M$. incana, the MiMYB1-MibHLH2-MiWDR1 complex activates the transcriptions of $M i F 3^{\prime} H, M i D F R$, and MiANS. Moreover, MibHLH1 probably enhances anthocyanin biosynthesis regulation using this complex (Fig. 6). The flower color diversity among $M$. incana cultivars involves differences not only in the anthocyanin component but also in anthocyanin accumulation levels (Dole and Wilkins 2005; Tatsuzawa et al. 2012). However, determining the function of $M i b H L H 1$ would require further research. In future studies, this finding would also be useful to identify the mutated genes responsible for each flower color in $M$. incana. 
Fig. 6 Regulation of anthocyanin biosynthesis in Matthiola incana flower. The MiMYB1MibHLH2-MiWDR1 complex activates the transcriptions of $M i F 3^{\prime} H, M i D F R$, and MiANS as well as induces anthocyanin accumulation in $M$. incana petals. Moreover, MibHLH1 might function as an enhancer for anthocyanin biosynthesis when it coexists with the MiMYB1-MibHLH2MiWDR1 complex. CHS, chalcone synthase; $\mathrm{CHI}$, chalcone isomerase; $\mathrm{F} 3 \mathrm{H}$, flavanone 3-hydroxylase; F3'H, flavonoid 3'-hydroxylase; DFR, dihydroflavonol 4-reductase; ANS, anthocyanidin synthase; 3GT, UDP-glucose:anthocyanidin 3-O-glucosyltransferase

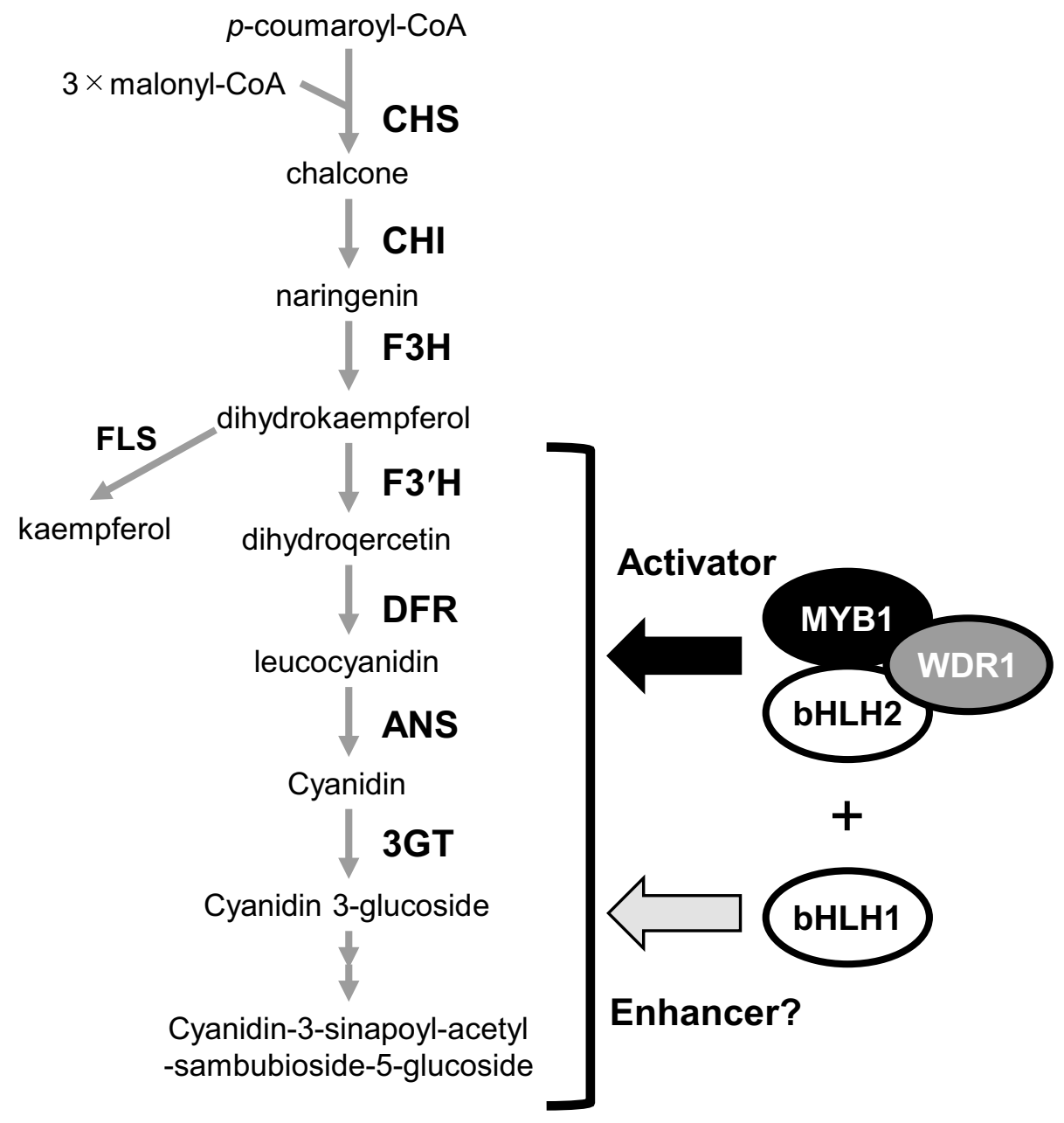

Author contribution statement TN conceived and designed research. $\mathrm{LN}$ and $\mathrm{TN}$ conducted quantitative expression analysis. LN, FT, and TN conducted flavonoid analysis. KK and TN conducted next-generation sequencing data analysis. YA, KK, KT, VA, and JAD conducted transient expression analysis using virus vectors. MO and KS discussed and revised the manuscript. $\mathrm{LN}$ and $\mathrm{TN}$ wrote the manuscript. All authors read and approved the manuscript.

Acknowledgements We thank Ms. Nobue Nakamura, Shizuoka University, for technical support. This work was supported by JSPS KAKENHI Grant Number 17H03765 and Ministerio de Ciencia, Innovación y Universidades (Spain; co-financed European Union FEDER funds) Grant BIO2017-83184-R.

\section{Compliance with ethical standards}

Conflict of interest The authors declare that they have no conflict of interest.

\section{References}

Allan AC, Espley RV (2018) MYBs drive novel consumer traits in fruits and vegetables. Trends Plant Sci 23:693-705. https://doi. org/10.1016/j.tplants.2018.06.001

Bedoya LC, Martinez F, Orzaez D, Daros JA (2012) Visual tracking of plant virus infection and movement using a reporter MYB transcription factor that activates anthocyanin biosynthesis. Plant Physiol 158:1130-1138. https://doi.org/10.1104/pp.111.192922

Bernhardt C, Lee MM, Gonzalez A, Zhang F, Lloyd A, Schiefelbein J (2003) The bHLH genes GLABRA3 (GL3) and ENHANCER OF GLABRA3 (EGL3) specify epidermal cell fate in the Arabidopsis root. Development 130:6431-6439. https://doi.org/10.1242/ dev.00880

Chen D, Liu Y, Pan Q, Li FF, Zhang Q, Ge X, Li Z (2018) De novo transcriptome assembly, gene expressions and metabolites for flower color variation of two garden species in Brassicaceae. Sci Hortic 240:592-602. https://doi.org/10.1016/j.scienta.2018.06.057

Chiu LW, Zhou X, Burke S, Wu X, Prior RL, Li L (2010) The purple cauliflower arises from activation of a MYB transcription factor. Plant Physiol 154:1470-1480. https://doi.org/10.1104/ pp. 110.164160

Crane MB, Lawrence MB (1947) The genetics of garden plants. Macmillan \& Co. Ltd, London, pp 50-54

Dole JM, Wilkins HF (2005) Matthiola. Floriculture prinples and species. Pearson Prentice Hall, New Jersey, pp 682-687 
Dressel A, Hemleben V (2009) Transparent Testa Glabra 1 (TTG1) and TTG1-like genes in Matthiola incana $\mathrm{R}$. Br. and related Brassicaceae and mutation in the WD-40 motif. Plant Biol 11:204-212. https://doi.org/10.1111/j.1438-8677.2008.00099.x

Forkmann G (1980) The B-ring hydroxylation pattern of intermediates of anthocyanin synthesis in pelargonidin- and cyanidin-producing lines of Matthiola incana. Planta 148:157-161. https://doi. org/10.1007/BF00386416

Forkmann G (1993) Genetics of flavonoids. In: Harborn JB (ed) The flavonoids: advances in research scince 1986. Chapman \& Hall, London, pp 537-564

Grabherr MG, Haas BJ, Yassour M, Levin JZ, Thompson DA, Amit I, Adiconis X, Fan L, Raychowdhury R, Zeng Q, Chen Z, Mauceli E, Hacohen N, Gnirke A, Rhind N, di Palma F, Birren BW, Nusbaum C, Lindblad-Toh K, Friedman N, Regev A (2011) Full-length transcriptome assembly from RNA-seq data without a reference genome. Nat Biotechnol 29:644-652. https://doi. org/10.1038/nbt.1883

Hemleben V, Dressel A, Epping B, Lukacin R, Martens S, Austin M (2004) Characterization and structural features of a chalcone synthase mutation in a white-flowering line of Matthiola incana R. Br. (Brassicaceae). Plant Mol Biol 55:455-465. https://doi. org/10.1007/s11103-004-1125-y

Hisamatsu T, Koshioka M, Kubota S, Fujime Y, King RW, Mander LN (2000) The role of gibberellin biosynthesis in the control of growth and flowering in Matthiola incana. Physiol Plant 109:97-105. https://doi.org/10.1034/j.1399-3054.2000.10011 4.x

Kamada K, Omata S, Yamagishi N, Kasajima I, Yoshikawa N (2018) Gentian (Gentiana triflora) prevents transmission of apple latent spherical virus (ALSV) vector to progeny seeds. Planta 248:14311441. https://doi.org/10.1007/s00425-018-2992-9

Li X, Chen L, Hong M, Zhang Y, Zu F, Wen J, Yi B, Ma C, Shen J, Tu J, Fu T (2012) A large insertion in bHLH transcription factor BrTT8 resulting in yellow seed coat in Brassica rapa. PLoS ONE 7:e44145. https://doi.org/10.1371/journal.pone.0044145

Lim SH, Song JH, Kim DH, Kim JK, Lee JY, Kim YM, Ha SH (2016) Activation of anthocyanin biosynthesis by expression of the radish R2R3-MYB transcription factor gene RsMYB1. Plant Cell Rep 35:641-653. https://doi.org/10.1007/s00299-015-1909-3

Lim SH, Kim DH, Kim JK, Lee JY, Ha SH (2017) A radish basic Helix-Loop-Helix transcription factor, RsTT8 acts a positive regulator for anthocyanin biosynthesis. Front Plant Sci 8:1917. https ://doi.org/10.3389/fpls.2017.01917

Llorente B, Torres-Montilla S, Morelli L, Florez-Sarasa I, Ezquerro M, D'andrea L, Majer E, Troncoso A, Fernie AR, Daròs JA, Rodriguez-Concepcion M (2019) Synthetic biogenesis of chromoplasts from leaf chloroplasts. bioRxive 819177. https://doi. org/10.1101/819177

Mortimer CL, Dugdale B, Dale JL (2015) Updates in inducible transgene expression using viral vectors: from transient to stable expression. Curr Opin Biotechnol 32:85-92. https://doi. org/10.1016/j.copbio.2014.11.009

Nakatsuka T, Koishi K (2018) Molecular characterization of a doubleflower mutation in Matthiola incana. Plant Sci 268:39-46. https ://doi.org/10.1016/j.plantsci.2017.12.009

Nakatsuka T, Nishihara M, Mishiba K, Yamamura S (2005) Temporal expression of flavonoid biosynthesis-related genes regulates flower pigmentation in gentian plants. Plant Sci 168:1309-1318. https://doi.org/10.1016/j.plantsci.2005.01.009

Nakatsuka T, Haruta KS, Pitaksutheepong C, Abe Y, Kakizaki Y, Yamamoto K, Shimada N, Yamamura S, Nishihara M (2008) Identification and characterization of R2R3-MYB and bHLH transcription factors regulating anthocyanin biosynthesis in gentian flowers. Plant Cell Physiol 49:1818-1829. https://doi.org/10.1093/ pcp/pen 163
Nakatsuka T, Sasaki N, Nishihara M (2014) Transcriptional regulators of flavonoid biosynthesis and their application to flower color modification in Japanese gentians. Plant Biotechnol 31:389-399. https://doi.org/10.5511/plantbiotechnology.14.0731a

Nakatsuka T, Suzuki T, Harada K, Kobayashi Y, Dohra H, Ohno H (2019) Floral organ- and temperature-dependent regulation of anthocyanin biosynthesis in Cymbidium hybrid flowers. Plant Sci 287:110173. https://doi.org/10.1016/j.plantsci.2019.110173

Nesi N, Jond C, Debeaujon I, Caboche M, Lepiniec L (2001) The Arabidopsis TT2 gene encodes an R2R3 MYB domain protein that acts as a key determinant for proanthocyanidin accumulation in developing seed. Plant Cell 13:2099-2114. https://doi. org/10.1105/tpc.010098

Quattrocchio F, Wing JF, Leppen H, Mol J, Koes RE (1993) Regulatory genes controlling anthocyanin pigmentation are functionally conserved among plant species and have distinct sets of target genes. Plant Cell 5:1497-1512. https://doi.org/10.1105/tpc.5.11.1497

Rail S, Hemleben V (1984) Characterization and expression of chalcone synthase in different genotypes of Matthiola incana $\mathrm{R} . \mathrm{Br}$. during flower development. Plant Mol Biol 3:137-145. https://doi. org/10.1007/BF00016061

Ramsay NA, Walker AR, Mooney M, Gray JC (2003) Two basichelix-loop-helix genes (MYC-146 and GL3) from Arabidopsis can activate anthocyanin biosynthesis in a white-flowered Matthiola incana mutant. Plant Mol Biol 52:679-688. https://doi. org/10.1023/a:1024852021124

Saito N, Tatsuzawa F, Nishiyama A, Yokoi M, Shigihara A, Honda $\mathrm{T}$ (1995) Acylated cyanidin 3-sambubioside-5-glucosides in Matthiola incana. Phytochemistry 38:1027-1032. https://doi. org/10.1016/j.phytochem.2006.05.001

Saito K, Yonekura-Sakakibara K, Nakabayashi R, Higashi Y, Yamazaki M, Tohge T, Fernie AR (2013) The flavonoid biosynthetic pathway in Arabidopsis: structural and genetic diversity. Plant Physiol Biochem 72:21-34. https://doi.org/10.1016/j.plaphy.2013.02.001

Sánchez F, Martinez-Herrera D, Aguilar I, Ponz F (1998) Infectivity of turnip mosaic potyvirus cDNA clones and transcripts on the systemic host Arabidopsis thaliana and local lesion hosts. Virus Res 55:207-219. https://doi.org/10.1016/S0168-1702(98)00049-5

Sasaki K, Mitsuda N, Nashima K, Kishimoto K, Katayose Y, Kanamori H, Ohmiya A (2017) Generation of expressed sequence tags for discovery of genes responsible for floral traits of Chrysanthemum morifolium by next-generation sequencing technology. BMC Genomics 18:683. https://doi.org/10.1186/s12864-017-4061-3

Saunders ER (1928) Matthiola. Bibliographia. Genetica 4:141-170

Seyffert W (1971) Simulation of quantitative characters by genes with biochemically definable action. Theoret Appl Genetics 41:285291. https://doi.org/10.1007/BF00277333

Spribille R, Forkmann G (1981) Genetic control of chalcone synthase activity in flowers of Matthiola incana R. Br. Z Naturforsch 36C:619-624

Suzuki K, Suzuki T, Nakatsuka T, Dohra H, Yamagishi M, Matsuyama K, Matsuura H (2016) RNA-seq-based evaluation of bicolor tepal pigmentation in Asiatic hybrid lilies (Lilium spp.). BMC Genomics 17:611. https://doi.org/10.1186/s12864-016-2995-5

Tatsuzawa F, Saito N, Toki K, Shinoda K, Honda T (2012) Flower colors and their anthocyanins in Matthiola incana cultivars (Brassicaceae). J Jpn Soc Hort Sci 81:91-100. https://doi.org/10.2503/ jjshs1.81.91

Tatsuzawa F, Okuyama N, Kato K, Shono H, Takeda J, Kofujita H (2014) Sinapoylglucoside and kaempferol glycosides in flowers of Matthiola incana cultivars (Brassicaceae). Hort Res (Japan) 13:85-89. https://doi.org/10.2503/hrj.13.85. (In Japanese with English abstract)

Teusch M, Forkmann G, Seyffert W (1986) Genetic control of UDP-glucose: anthocyanin 5-O-glucosyltransferase from 
flowers of Matthiola incana R.Br. Planta 168:586-591. https:// doi.org/10.1007/BF00392280

Teusch M, Forkmann G, Seyffert W (1987) Genetic control of hydroxycinnamoyl-coenzyme a: anthocyanidin 3-glycoside-hydroxycinnamoyltransferase from petals of Matthiola incana. Phytochemistry 26:991-994. https://doi.org/10.1016/S0031-9422(00)82333-X

Thole V, Worland B, Snape JW, Vain P (2007) The pCLEAN dual binary vector system for Agrobacterium-mediated plant transformation. Plant Physiol 145:1211-1219. https://doi.org/10.1104/ pp.107.108563

Tian J, Pei H, Zhang S, Chen J, Chen W, Yang R, Meng Y, You J, Gao J, Ma N (2014) TRV-GFP: a modified Tobacco rattle virus vector for efficient and visualizable analysis of gene function. J Exp Bot 65:311-322. https://doi.org/10.1093/jxb/ert381

Wei YL, Li JN, Lu J, Tang ZL, Pu DC, Chai YR (2007) Molecular cloning of Brassica napus TRANSPARENT TESTA 2 gene family encoding potential MYB regulatory proteins of proanthocyanidin biosynthesis. Mol Biol Rep 34:105-120. https://doi.org/10.1007/ s11033-006-9024-8

Xu W, Dubos C, Lepiniec L (2015) Transcriptional control of flavonoid biosynthesis by MYB-bHLH-WDR complexes. Trends Plant Sci 20:176-185. https://doi.org/10.1016/j.tplants.2014.12.001

Yang X, Lu Y, Zhao X, Jiang L, Xu S, Peng J, Zheng H, Lin L, Wu Y, MacFarlane S, Chen J, Yan F (2019) Downregulation of nuclear protein $\mathrm{H} 2 \mathrm{~B}$ induces salicylic acid mediated defense against $\mathrm{PVX}$ infection in Nicotiana benthamiana. Front Microbiol 10:1000. https://doi.org/10.3389/fmicb.2019.01000

Yuan Y, Chiu LW, Li L (2009) Transcriptional regulation of anthocyanin biosynthesis in red cabbage. Planta 230:1141-1153. https:// doi.org/10.1007/s00425-009-1013-4

Zhang F, Gonzalez A, Zhao M, Payne CT, Lloyd A (2003) A network of redundant bHLH proteins functions in all TTG1-dependent pathways of Arabidopsis. Development 130:4859-4869. https:// doi.org/10.1242/dev.00681

Zhang B, Hu Z, Zhang Y, Li Y, Zhou S, Chen G (2012) A putative functional MYB transcription factor induced by low temperature regulates anthocyanin biosynthesis in purple kale (Brassica Oleracea var. acephala f. tricolor). Plant Cell Rep 31:281-289. https://doi.org/10.1007/s00299-011-1162-3

Zhao D, Jiang Y, Ning C, Meng J, Lin S, Ding W, Tao J (2014) Transcriptome sequencing of a chimaera reveals coordinated expression of anthocyanin biosynthetic genes mediating yellow formation in herbaceous peony (Paeonia lactiflora Pall.). BMC Genomics 15:689. https://doi.org/10.1186/1471-2164-15-689

Publisher's Note Springer Nature remains neutral with regard to jurisdictional claims in published maps and institutional affiliations. 